\author{
ANDRZEJ KRAJKA, ZDZISEAW RYCHLIK \\ and JOANNA WASIURA-MAŚLANY
}

\title{
On the almost sure convergence of randomly indexed maximum of random variables
}

\author{
Dedicated to Professor Yuri Kozitsky on the occasion of his 70th birthday
}

\begin{abstract}
We prove an almost sure random version of a maximum limit theorem, using logarithmic means for $\max _{1 \leq i \leq N_{n}} X_{i}$, where $\left\{X_{n}, n \geq 1\right\}$ is a sequence of identically distributed random variables and $\left\{N_{n}, n \geq 1\right\}$ is a sequence of positive integer random variables independent of $\left\{X_{n}, n \geq 1\right\}$. Furthermore, we consider the almost sure random version of a limit theorem for $k$ th order statistics.
\end{abstract}

\section{Introduction}

Let $\left\{X_{n}, n \geq 1\right\}$ be a sequence of independent and identically distributed random variables with $E X_{1}=0, E X_{1}^{2}=1$, and let $S_{n}=X_{1}+X_{2}+\cdots+X_{n}$. The almost sure central limit theorem (ASCLT) says that for any fixed $x \in \Re$ we have

$$
\lim _{n \rightarrow \infty} \frac{1}{\log n} \sum_{j=1}^{n} \frac{1}{j} I\left[\frac{S_{j}}{\sqrt{j}} \leq x\right]=\Phi(x), \text { a.s., }
$$

where $\Phi(x)$ denotes the standard normal distribution function. This result is a generalization of the arcsin law of Andersen and was firstly obtained by

2010 Mathematics Subject Classification. Primary: 60F05; Secondary: 60F15, 60G70.

Key words and phrases. Almost sure central limit theorem, randomly indexed sums. 
Brosamler [4] and Schatte [19] under additional moment conditions on $X_{1}$ and by Lacey and Philipp [13] under assuming only finite variance. This result is probably the most intensively investigated in the last decade. For the different generalizations of (1) cf. [3].

Let us consider the following three sets of distribution functions:

Case (i) $\mathcal{D}_{1}=\{F \in \mathcal{L}$ : there exists the positive function $g$ such that

$$
\left.\frac{1-F(t+x g(t))}{1-F(t)} \longrightarrow e^{-x} \text {, as } t \rightarrow x_{F} \text {, for all } x \in \Re\right\} \text {, }
$$

Case (ii) $\mathcal{D}_{2, \alpha}=\left\{F \in \mathcal{L}: x_{F}=\infty\right.$ and $\frac{1-F(t x))}{1-F(t)} \longrightarrow x^{-\alpha}$, as $t \rightarrow \infty$, for all $x>0\}$, for some $\alpha>0$,

Case (iii) $\mathcal{D}_{3, \alpha}=\left\{F \in \mathcal{L}: x_{F}<\infty\right.$ and $\frac{1-F\left(x_{F}-x h\right)}{1-F\left(x_{F}-h\right)} \longrightarrow x^{\alpha}$, as $h \rightarrow 0_{+}$, for all $\left.x>0\right\}$, for some $\alpha>0$,

where $F($.$) denotes the distribution function of X_{1}, x_{F}=\inf \{x: F(x)=1\}$, and $\mathcal{L}$ denotes the set of distribution functions on $\Re$. It is known (cf. [14, 17]) that if $F$ belongs to $D_{1}, D_{2, \alpha}$ or $D_{3, \alpha}$ with some $\alpha>0$, then there exist constants $\left\{a_{n}, b_{n}, n \geq 1\right\}$ such that

$$
a_{n}\left(\max _{1 \leq j \leq n} X_{j}-b_{n}\right) \stackrel{\mathcal{D}}{\longrightarrow} G, \text { as } n \rightarrow \infty,
$$

where $G$ is equal to

$$
\begin{gathered}
G_{1}(x)=e^{-e^{-x}}, \\
G_{2, \alpha}(x)= \begin{cases}0, & x \leq 0, \\
e^{-x^{-\alpha}}, & x>0,\end{cases}
\end{gathered}
$$

or

$$
G_{3, \alpha}(x)= \begin{cases}e^{-(-x)^{\alpha}}, & x \leq 0, \\ 1, & \$ x>0,\end{cases}
$$

respectively. Conversely, if (2) holds for some sequence of independent and identically distributed random variables $\left\{X_{n}, n \geq 1\right\}$, then the possible nondegenerate limits $G$ are $G_{1}, G_{2, \alpha}$, or $G_{3, \alpha}$ only. Furthermore, under assumption (2) we have

$$
a_{n}\left(X_{n-k: n}-b_{n}\right) \stackrel{\mathcal{D}}{\longrightarrow} G(x) \sum_{t=0}^{k} \frac{(-\log G(x))^{t}}{t !}, \text { as } n \rightarrow \infty,
$$

where by $X_{1: n} \leq X_{2: n} \leq X_{3: n} \leq \cdots \leq X_{n: n}$ we denote the order statistics of $\left\{X_{1}, X_{2}, \ldots, X_{n}\right\}$. These results are called the max limit theorems. In 1998, Fahrner I. and Stadtmüller V. [8], and independently Cheng S., Peng L. and Qi Y. [6] proved the max limit schema version of ASCLT with $k=0$ (cf. [7], 
too). They proved that if (2) holds, then

$$
\lim _{n \rightarrow \infty} \frac{1}{\log n} \sum_{j=1}^{n} \frac{1}{j} I\left[a_{j}\left(\max _{1 \leq i \leq j} X_{i}-b_{j}\right) \leq x\right]=G(x), \text { a.s. }
$$

for any $x \in \Re$. This result was generalized on case $k$ th order statististic by Stadtmüller [18]. Assuming (2) and that $\left\{X_{n}, n \geq 1\right\}$ is an independent and identically distributed sequence with continuous distribution function of $X_{1}$, he proved that

(5) $\lim _{n \rightarrow \infty} \frac{1}{\log n} \sum_{j=1}^{n} \frac{1}{j} I\left[a_{j}\left(X_{j-k: j}-b_{j}\right) \leq x\right]=G(x) \sum_{t=0}^{k} \frac{(-\log G(x))^{t}}{t !}$, a.s.

(In order to get simpler formulas here and in what follows, we put $P\left[X_{j: k} \leq\right.$ $x]:=1$ for $j \leq 0, k \geq 0$ or $k>j$.) However, among the different generalizations of ASCLT there is no version of ASCLT with random indices, although the first central limit theorem results and max limit theorem results almost at once obtained such generalization (cf., for e.g., [16], in CLT case and [1] in max limit theorem case). The main reason is that the random indexing introduces the big level of complications and numerical difficulties. In this paper we generalize the results of $[6,8]$ and $[18]$ in the following directions:

(i) We drop the assumption of interindependency of $\left\{X_{n}, n \geq 1\right\}$ considering the stationary sequences.

(ii) We consider the randomly indexed version of (5). Assuming independence between the sequence $\left\{X_{n}, n \geq 1\right\}$ and the sequence of random indices $\left\{N_{n}, n \geq 1\right\}$, we give the conditions under which

(6)

$$
\begin{aligned}
\lim _{n \rightarrow \infty} \frac{1}{\log n} \sum_{j=1}^{n} \frac{1}{j}\left(I\left[a_{j}\left(X_{N_{j}-k: N_{j}}-b_{j}\right) \leq x\right]\right. \\
\left.-G^{\frac{N_{j}}{j}}(x) \sum_{t=0}^{k} \frac{1}{t !}\left[-\frac{N_{j}}{j} \log G(x)\right]^{t}\right)=0 \text {, a.s. }
\end{aligned}
$$

(iii) In comparison with the result of [18], we omit the assumption on continuity of the distribution function of $X_{1}$.

In the whole paper we will use the notations: $x \vee y=\max \{x, y\}$ and $x \wedge y=\min \{x, y\}$.

\section{Main results}

Let $\left\{X_{n}, n \geq 1\right\}$ be a sequence of identically distributed random variables with the common distribution function $F$ such that for some constants $\left\{a_{n}, b_{n}, n \geq 1\right\}$ we have

$$
a_{n}\left(\max _{1 \leq j \leq n} X_{j}-b_{n}\right) \stackrel{\mathcal{D}}{\longrightarrow} G, \text { as } n \rightarrow \infty
$$


with $G$ equal to $G_{1}, G_{2, \alpha}$ or $G_{3, \alpha}$. Let $\left\{\tilde{X}_{n}, n \geq 1\right\}$ be a sequence of independent and identically distributed random variables with the distribution function $F$. For $x, y \in \Re$ we will put

$$
v_{j}=x / a_{j}+b_{j}, j \geq 1,
$$

and for some positive constants $\theta$ and positive integer $k$ :

$$
\begin{aligned}
\alpha_{j, h}(x, y) & =\left|P\left[X_{j-k: j} \leq x, X_{h-k: h} \leq y\right]-P\left[\tilde{X}_{j-k: j} \leq x, \tilde{X}_{h-k: h} \leq y\right]^{\theta}\right|, \\
\text { (8) } \alpha_{j}(x) & =\left|P\left[X_{j-k: j} \leq x\right]-P\left[\tilde{X}_{j-k: j} \leq x\right]^{\theta}\right| .
\end{aligned}
$$

The coefficients $\alpha_{j}(x)$ defined in (8) are called the extremal index of stationary sequence $\left\{X_{n}, n \geq 1\right\}$ and were introduced in [15] and studied intensively in [11]. These coefficients stand the analogue of mixing condition in max-limit theory.

Theorem 1. Let $\left\{X_{n}, n \geq 1\right\}$ be a sequence of identically distributed random variables with common distribution function $F$ satisfying condition (7) for some numbers $\left\{a_{n}, b_{n}, n \geq 1\right\}$. Let $\left\{N_{n}, n \geq 1\right\}$ be a sequence of pairwise independent random indexes independent of $\left\{X_{n}, n \geq 1\right\}$. Let us assume that for some fixed $\mu \in(0,1)$,

$$
\sum_{h=1}^{n} \sum_{j=1}^{h-1} \frac{1}{j h} E\left(\frac{N_{j} \wedge N_{h}}{h} \wedge 1\right)=O\left((\log n)^{2-\mu}\right) .
$$

Furthermore, let us assume that

$$
\sum_{h=1}^{n} \sum_{j=1}^{h-1} \frac{1}{j h} E \alpha_{N_{j}, N_{h}}\left(v_{j}, v_{h}\right)=O\left((\log n)^{2-\mu}\right),
$$

and

$$
\sum_{j=1}^{n} \frac{1}{j} E \alpha_{N_{j}}\left(v_{j}\right)=O\left((\log n)^{2-\mu}\right) .
$$

In the case when $G=G_{2, \alpha}$ with some $\alpha>0$, we assume additionally that for some $\delta_{0}>0$,

$$
P\left[\frac{N_{j}}{j}<\delta_{0}\right]=O\left((\log j)^{-\mu}\right) \text {, as } j \rightarrow \infty .
$$

Then

$$
\frac{1}{\log n} \sum_{j=1}^{n} \frac{1}{j}\left(I\left[a_{j}\left(X_{N_{j}-k: N_{j}}-b_{j}\right) \leq x\right]-H_{G, k, N_{j} / j}^{\theta}(x)\right) \stackrel{a . s .}{\longrightarrow} 0,
$$

as $n \rightarrow \infty$, where

$$
H_{G, k, \beta}(x)= \begin{cases}G^{\beta}(x) \sum_{t=0}^{k} \frac{1}{t !}[-\beta \log G(x)]^{t}, & \text { if } G(x)>0, \\ 0, & \text { if } G(x)=0 .\end{cases}
$$


Let $f($.$) be a a.e. continuous, bounded real function, such that f(-\infty)=0$, $f(+\infty)=0$. If $\liminf \frac{N_{j}}{j}>C>0$, then

(14) $\frac{1}{\log n} \sum_{j=1}^{n} \frac{1}{j}\left(f\left(a_{j}\left(X_{N_{j}-k: N_{j}}-b_{j}\right)\right)-\int_{-\infty}^{\infty} f(x) H_{G, k, N_{j} / j}^{\theta}(d x)\right) \stackrel{a . s .}{\longrightarrow} 0$,

as $n \rightarrow \infty$.

Additionally, if there exists a positive bounded from 0 random variable $\lambda$ such that $d\left(\frac{N_{j}}{j}, \lambda\right)=O\left((\log j)^{-\mu}\right)$, where $d(X, Y)$ is the Lévy-Prokhorov's distance between random variables $X$ and $Y$ (i.e. $d(X, Y)=\inf \{\epsilon>0$ : $P[|X-Y|>\epsilon]<\epsilon\})$, then

$$
\frac{1}{\log n} \sum_{j=1}^{n} \frac{1}{j}\left(f\left(a_{j}\left(X_{N_{j}-k: N_{j}}-b_{j}\right)\right)-\int_{-\infty}^{\infty} f(x) H_{G, k, \lambda}^{\theta}(d x)\right) \stackrel{a . s .}{\longrightarrow} 0,
$$

as $n \rightarrow \infty$.

Corollary 1. Let $\left\{X_{n}, n \geq 1\right\}$ be a sequence of independent and identically distributed random variables with common distribution function $F$, and let $\left\{N_{n}, n \geq 1\right\}$ be a sequence of pairwise independent random indexes independent of $\left\{X_{n}, n \geq 1\right\}$. Let us assume (7), (9), and in case when $G=G_{2, \alpha}$ with some $\alpha>0$, (12) hold. Then

$$
\frac{1}{\log n} \sum_{j=1}^{n} \frac{1}{j}\left(I\left[a_{j}\left(X_{N_{j}-k: N_{j}}-b_{j}\right) \leq x\right]-H_{G, k, N_{j} / j}(x)\right) \stackrel{a . s .}{\longrightarrow} 0,
$$

as $n \rightarrow \infty$.

Let $f($.$) be a a.e. continuous, bounded real function, such that f(-\infty)=0$, $f(+\infty)=0$. If $\liminf \frac{N_{j}}{j}>C>0$, then

$$
\frac{1}{\log n} \sum_{j=1}^{n} \frac{1}{j}\left(f\left(a_{j}\left(X_{N_{j}-k: N_{j}}-b_{j}\right)\right)-\int_{-\infty}^{\infty} f(x) H_{G, k, N_{j} / j}(d x)\right) \stackrel{a . s_{.}}{\longrightarrow} 0,
$$

as $n \rightarrow \infty$.

Additionally, if there exists a positive bounded from 0 random variable $\lambda$ such that $d\left(\frac{N_{j}}{j}, \lambda\right)=O\left((\log j)^{-\mu}\right)$, then

$$
\frac{1}{\log n} \sum_{j=1}^{n} \frac{1}{j}\left(f\left(a_{j}\left(X_{N_{j}-k: N_{j}}-b_{j}\right)\right)-\int_{-\infty}^{\infty} f(x) H_{G, k, \lambda}(d x)\right) \stackrel{a . s}{\longrightarrow} 0,
$$

as $n \rightarrow \infty$.

Corollary 2. Let $\left\{X_{n}, n \geq 1\right\}$ be a sequence of independent and identically distributed random variables with common distribution function $F$, and let $\left\{N_{n}, n \geq 1\right\}$ be a sequence of pairwise independent random indexes independent of $\left\{X_{n}, n \geq 1\right\}$. Let us assume (7), (9), and in case when $G=G_{2, \alpha}$ 
with some $\alpha>0$, (12) hold. Then

$$
\frac{1}{\log n} \sum_{j=1}^{n} \frac{1}{j}\left(I\left[a_{j}\left(\max _{1 \leq i \leq N_{j}} X_{i}-b_{j}\right) \leq x\right]-G^{N_{j} / j}(x)\right) \stackrel{a . s .}{\longrightarrow} 0,
$$

as $n \rightarrow \infty$.

Let $f($.$) be a a.e. continuous, bounded real function, such that f(-\infty)=0$, $f(+\infty)=0$. If $\liminf \frac{N_{j}}{j}>C>0$, then

$$
\frac{1}{\log n} \sum_{j=1}^{n} \frac{1}{j}\left(f\left(a_{j}\left(\max _{1 \leq i \leq N_{j}} X_{i}-b_{j}\right)\right)-\int_{-\infty}^{\infty} f(x) G^{N_{j} / j}(d x)\right) \stackrel{a . s_{.}}{\longrightarrow} 0,
$$

as $n \rightarrow \infty$.

Additionally, if there exists a positive bounded from 0 random variable $\lambda$ such that $d\left(\frac{N_{j}}{j}, \lambda\right)=O\left((\log j)^{-\mu}\right)$, then

$$
\frac{1}{\log n} \sum_{j=1}^{n} \frac{1}{j}\left(f\left(a_{j}\left(\max _{1 \leq i \leq N_{j}} X_{i}-b_{j}\right)\right)-\int_{-\infty}^{\infty} f(x) G^{\lambda}(d x)\right) \stackrel{a . s}{\longrightarrow} 0,
$$

as $n \rightarrow \infty$.

Putting in Corollary 1 and 2 the sequence $N_{j}=j$, a.s., $j \geq 1$, we obtain the main results in [6], [8] and [18].

\section{Proofs}

Lemma 1. Let $x, y \in[0,1], \alpha>0$ be arbitrary numbers.

(i) For $y>0$, we have

$$
\left|x^{\alpha}-y^{\alpha}\right| \leq \alpha|x-y|^{\alpha \wedge 1} .
$$

(ii) For $\alpha \leq 1$, we have

$$
\left|x^{\alpha}-y^{\alpha}\right| \leq \frac{|x-y|}{|y|^{2}} .
$$

(iii) For arbitrary $t \in \mathcal{N}, 1 / t>\alpha$, we have

$$
\left|x(-\log x)^{t}-y(-\log y)^{t}\right| \leq\left(\frac{e}{\alpha} \wedge t\right) \frac{(e / \alpha)^{t-1}}{1-\alpha t}\left|x^{1-\alpha t}-y^{1-\alpha t}\right| .
$$

Proof of Lemma 1. If $\alpha \leq 1$ we consider the functions $f(x)=x^{\alpha}-y^{\alpha}$ and $g(x)=(x-y)^{\alpha}$ in the interval $[y, 1]$. Now $f(y)=0=g(y)$ and inequality

$$
f^{\prime}(x)=\frac{\alpha}{x^{1-\alpha}} \leq \frac{\alpha}{(x-y)^{1-\alpha}}=g^{\prime}(x),
$$

ends the proof of (i) in the case $x>y$. Case $x<y$ follows by symmetry and case $x=y$ is obvious.

When $\alpha \in(0,1)$ let $\mathrm{k}$ be chosen such that $\frac{1}{2^{k}}<\alpha \leq \frac{1}{2^{k-1}}$. Then $\left(x^{2^{k} \alpha}-y^{2^{k} \alpha}\right)=\left(x^{\alpha}-y^{\alpha}\right)\left(x^{\alpha}+y^{\alpha}\right)\left(x^{2 \alpha}+y^{2 \alpha}\right)\left(x^{4 \alpha}+y^{4 \alpha}\right) \ldots\left(x^{2^{k-1} \alpha}+y^{2^{k-1} \alpha}\right)$. 
Thus, by the above proved case $\alpha>1$, we have

$$
|x-y| \geq\left|x^{\alpha}-y^{\alpha}\right| y^{\alpha\left(1+2+4+\cdots+2^{k-1}\right)}=\left|x^{\alpha}-y^{\alpha}\right| y^{\alpha\left(2^{k}-1\right)} \geq\left|x^{\alpha}-y^{\alpha}\right| y^{2-\alpha},
$$

which gives (ii).

For proof of Lemma 1 (iii) we consider the case that $x \geq y>0$ and $x(-\log x)^{t} \geq y(-\log y)^{t}$, firstly. Let us define two functions

$$
f(x)=x(-\log x)^{t}-y(-\log y)^{t} \text { and } g(x)=\frac{(e / \alpha)^{t}}{1-\alpha t}\left(x^{1-\alpha t}-y^{1-\alpha t}\right) .
$$

Obviously $f(y)=g(y)=0$ and

$$
f^{\prime}(x)=(-\log x)^{t-1}(-\log x-t) \leq(-\log x)^{t}, \quad g^{\prime}(x)=(e / \alpha)^{t} x^{-\alpha t} .
$$

Now we remark that the maximum of the function $-x^{\alpha} \log x$ in area $(0,+\infty)$ is achieved for $x=e^{-1 / \alpha}$ and is equal $e / \alpha$, which ends the proof of Lemma 1 (iii) in this case.

When $x \geq y>0$ and $x(-\log x)^{t} \leq y(-\log y)^{t}$, then $-t \leq \log x \leq 0$ (note that function $x(-\log x)^{t}$ is increasing in the interval $\left(0, e^{-t}\right)$ ). Thus, putting $f(x)=-x(-\log x)^{t}+y(-\log y)^{t}$, and $g(x)=t \frac{(e / \alpha)^{t-1}}{1-\alpha t}\left(x^{1-\alpha t}-\right.$ $\left.y^{1-\alpha t}\right)$, we have $f(y)=g(y)=0$ and $f^{\prime}(x)=(-\log x)^{t-1}(\log x+t) \leq$ $t(-\log x)^{t-1}, g^{\prime}(x)=t(e / \alpha)^{t-1} x^{-\alpha t}$, such that the argumentation similar to the above ends the proof.

In the paper [12] (Lemma 7) the following lemma was proved.

\section{Lemma 2.}

(a) Let $\left\{X_{n}, n \geq 1\right\}$ be a sequence of random variables such that $X_{n} \rightarrow$ 0 , a.s., as $n \rightarrow \infty$, and for some positive real constant $K$ and every $n,\left|X_{n}\right|<K$, a.s. Then

$$
\frac{1}{\log n} \sum_{j=1}^{n} \frac{X_{j}}{j} \stackrel{a . s .}{\longrightarrow} 0, \quad \text { as } n \rightarrow \infty .
$$

(b) Let $\left\{X_{n}, n \geq 1\right\}$ be an arbitrary sequence of random variables such that for some $\mu \in(0,1)$, we have $d\left(X_{n}, 0\right)=O\left((\log n)^{-\mu}\right)$ and $\left|X_{n}\right|<K$ a.s. for some positive constants $K$. Then

$$
\frac{1}{\log n} \sum_{j=1}^{n} \frac{X_{j}}{j} \stackrel{a . s .}{\longrightarrow} 0, \text { as } n \rightarrow \infty .
$$

(c) For every convergent to zero sequence of real numbers $\left\{\epsilon_{n}, n \geq 1\right\}$, we have

$$
\frac{1}{\log n} \sum_{j=1}^{n} \frac{\epsilon_{j}}{j} \longrightarrow 0, \text { as } n \rightarrow \infty .
$$


Lemma 3. Let $\left\{X_{n}, n \geq 1\right\}$ be a sequence of independent and identically distributed random variables such that $\mathcal{L}\left(X_{1}\right)=F($.$) . Then for every posi-$ tive integers $j, l, k$ such that $j \wedge l \geq k$, we have

$$
P\left[X_{j-k: j} \leq x\right]=\sum_{t=0}^{k}\left(\begin{array}{l}
j \\
t
\end{array}\right) F^{j-t}(x)(1-F(x))^{t},
$$

and

$$
P\left[X_{j-k: j} \leq x, X_{l-k: l} \leq y\right] \leq P\left[X_{j-k: j} \leq x\right] P\left[X_{l-k: l} \leq y\right] F(x \vee y)^{-j \wedge l} .
$$

Proof of Lemma 3. The evaluation (16) is proved in Lemma A.1 whereas the evaluation (17) is a small generalization of Lemma A.2 ([18], p. 422424). From Lemma A.2 in case $1 \leq k \leq j \leq l$ and $x \geq y$ and inequality $P\left[X_{j-k: j} \leq x\right] \leq P\left[X_{j: j} \leq x\right]=F^{j}(x)$, we have

$$
\begin{aligned}
P\left[X_{j-k: j} \leq x, X_{l-k: l} \leq y\right] & =P\left[X_{l-k: l} \leq y\right] \\
& \leq P\left[X_{j-k: j} \leq x\right] P\left[X_{l-k: l} \leq y\right] F(x \vee y)^{-j} .
\end{aligned}
$$

Proof of Theorem 1. In the whole proof we will use notation $\xi_{l, j}(k)=$ $I\left[a_{j}\left(X_{l-k: l}-b_{j}\right) \leq x\right], k \geq 0$. We have

$$
\begin{aligned}
& \frac{1}{\log n} \sum_{j=1}^{n} \frac{1}{j}\left(I\left[a_{j}\left(X_{N_{j}-k: N_{j}}-b_{j}\right) \leq x\right]-H_{G, k, N_{j} / j}^{\theta}(x)\right) \\
& =\frac{1}{\log n} \sum_{j=1}^{n} \frac{1}{j} \sum_{l=1}^{\infty}\left(\xi_{l, j}(k)-E \xi_{l, j}(k)\right) I\left[N_{j}=l\right] \\
& \quad+\frac{1}{\log n} \sum_{j=1}^{n} \frac{1}{j} \sum_{l=1}^{\infty}\left(E \xi_{l, j}(k)-H_{G, k, l / j}^{\theta}(x)\right) I\left[N_{j}=l\right] \\
& =V_{1}(n)+V_{2}(n), \text { say. }
\end{aligned}
$$

Step 1. At first we consider the case $G(x)>0$.

In order to prove that $\left|V_{1}(n)\right| \stackrel{\text { a.s. }}{\longrightarrow} 0$, we need some upper estimation on the value $\operatorname{cov}\left(\xi_{h, j}(k), \xi_{l, i}(k)\right)$. However, firstly we evaluate

$I_{h, j ; l, i}(k ; \theta)=P^{\theta}\left[\tilde{X}_{h-k: h} \leq v_{j}, \tilde{X}_{l-k: l} \leq v_{i}\right]-P^{\theta}\left[\tilde{X}_{h-k: h} \leq v_{j}\right] P^{\theta}\left[\tilde{X}_{l-k: l} \leq v_{i}\right]$.

By Lemma 3 and the fact that $F$ is nondecreasing and $v_{i \vee j} \leq v_{i} \vee v_{j}$ we have

$$
I_{h, j ; l, i}(k ; \theta) \leq\left|F^{-\theta(h \wedge l)}\left(v_{j \vee i}\right)-1\right| \wedge 1 .
$$

Now we consider the sequence $c_{h}=h\left(1-F\left(v_{h}\right)\right)$. By (2) and Theorem 1.5.1 in Leadbetter [14] we have for $x \in R$, and $h \rightarrow \infty$,

$$
c_{h} \rightarrow-\log G(x) \text {. }
$$


Since $\lim _{h \rightarrow \infty} 1-F\left(v_{h}\right)=\lim _{h \rightarrow \infty} \frac{-\log (G(x))}{h}=0$, then we may choose $n_{o}$ such that $1-F\left(v_{h}\right) \leq \frac{1}{4}$, for every $h \geq n_{o}$. Thus

$$
I_{h, j ; l, i}(k ; \theta) \leq\left|\left(1-\frac{(i \vee j)\left(1-F\left(v_{(i \vee j)}\right)\right)}{i \vee j}\right)^{-\theta(l \wedge h)}-1\right| \wedge 1 .
$$

From inequalities $e^{-2 x} \leq 1-x$ (valid for $0 \leq x \leq \frac{1}{4}$ ) and $\left|1-e^{x}\right| \leq|x| e^{|x|}$ we have for $i \vee j>n_{o}$,

$$
\begin{aligned}
I_{h, j ; l, i}(k ; \theta) & \leq\left|e^{\theta \frac{2 c(i \vee j)}{i \vee j}(l \wedge h)}-1\right| \wedge 1 \leq \frac{2 \theta c_{(i \vee j)}}{i \vee j}(l \wedge h) e^{\frac{2 \theta c(i \vee j)}{(i \vee j)}(l \wedge h)} \wedge 1 \\
& \leq \frac{2 e \theta c_{(i \vee j)}(l \wedge h)}{i \vee j} \wedge 1,
\end{aligned}
$$

and for $i \vee j \leq n_{o}$,

$$
I_{h, j ; l, i}(k ; \theta) \leq 1
$$

On the other hand, by $(8)$

$P\left[X_{h-k: h} \leq x\right] P\left[X_{l-k: l} \leq y\right]-P\left[\tilde{X}_{h-k: h} \leq x\right]^{\theta} P\left[\tilde{X}_{l-k: l} \leq y\right]^{\theta} \leq \alpha_{h}(x)+\alpha_{l}(y)$, thus in the case $h \leq l$, and $v_{j} \leq v_{i}$

$$
\operatorname{cov}\left(\xi_{h, j}(k), \xi_{l, i}(k)\right) \leq \alpha_{h, l}\left(v_{j}, v_{i}\right)+I_{h, j ; l, i}(k ; \theta)+\alpha_{h}\left(v_{j}\right)+\alpha_{l}\left(v_{i}\right),
$$

whereas in the case $h \leq l$, and $v_{j}>v_{i}$

$$
\operatorname{cov}\left(\xi_{h, j}(k), \xi_{l, i}(k)\right) \leq I_{h, j ; l, i}(k ; \theta)+\alpha_{h}\left(v_{j}\right)+2 \alpha_{l}\left(v_{i}\right) .
$$

Thus from (9)-(11) and (18)-(20)

$$
\begin{aligned}
\operatorname{Var}( & \left.V_{1}(n) \log n\right)=\sum_{\left\{l_{j} \in N^{N}\right\}} P\left[N_{j}=l_{j}, j \geq 1\right] \operatorname{Var}\left(\sum_{h=1}^{n} \frac{\xi_{l_{h}, h}}{h}\right) \\
\leq & 2 \sum_{h=1}^{n} \sum_{j=1}^{h-1} \frac{1}{j h} E \alpha_{N_{j}, N_{h}}\left(v_{j}, v_{h}\right)+2 \sum_{h=1}^{n} \sum_{j=1}^{h-1} \frac{\theta}{j h} E\left(\frac{N_{j} \wedge N_{h}}{h} \wedge 1\right) \\
& +4 \sum_{h=1}^{n} \frac{1}{h} E \alpha_{N_{h}}\left(v_{h}\right) \log h+\log n+\log ^{2} n_{o}=O\left((\log n)^{2-\mu}\right) .
\end{aligned}
$$

Now we put $n=n(k)=2^{k^{2 / \mu}}$ and by Chebyshev's inequality and BorelCantelli lemma, we have

$$
V_{1}(n(k)) \longrightarrow 0, \text { as } k \rightarrow \infty,
$$

with probability one. Furthermore, for $n(k)<n<n(k+1)$

$$
V_{1}(n)=\frac{\log n(k)}{\log n} V_{1}(n(k))+\frac{1}{\log n} \sum_{j=n(k)+1}^{n} \frac{1}{j} \sum_{l=1}^{\infty}\left(\xi_{l, j}(k)-E \xi_{l, j}(k)\right) I\left[N_{j}=l\right] .
$$


Taking into account $\left(\frac{k}{1+k}\right)^{2 / \mu} V_{1}(n(k)) \leq \frac{\log n(k)}{\log n} V_{1}(n(k)) \leq V_{1}(n(k)),(21)$ and evaluation

$$
\frac{1}{\log n} \sum_{j=n(k)+1}^{n} \frac{1}{j} \leq \frac{\log \frac{n(k+1)}{n(k)}}{\log n(k)}=C \frac{(k+1)^{2 / \mu}}{k^{2 / \mu}} \rightarrow 0, \text { as } k \rightarrow \infty,
$$

we get

$$
\lim _{n \rightarrow \infty} V_{1}(n)=0 \quad \text { a.s. }
$$

Let us prove $V_{2}(n) \stackrel{\text { a.s. }}{\longrightarrow} 0$. We have by Lemma 1

$$
\begin{aligned}
& \left|V_{2}(n)\right| \leq \frac{1}{\log n} \sum_{j=1}^{n} \frac{1}{j} \sum_{l=1}^{\infty}\left|P\left[X_{l-k: l} \leq v_{j}\right]-P^{\theta}\left[\tilde{X}_{l-k: l} \leq v_{j}\right]\right| I\left[N_{j}=l\right] \\
& \quad+\frac{1}{\log n} \sum_{j=1}^{n} \frac{1}{j} \sum_{l=1}^{\infty}\left|P\left[\tilde{X}_{l-k: l} \leq v_{j}\right]-H_{G, k, l / n}(x)\right|^{\theta \wedge 1} I\left[N_{j}=l\right] \\
& \leq \frac{1}{\log n} \sum_{j=1}^{n} \frac{1}{j} E \alpha_{N_{j}}\left(v_{j}\right)+\frac{1}{\log n} \sum_{j=1}^{n} \frac{1}{j} \sum_{l=1}^{\infty} k^{\theta \wedge 1}\left(\max _{0 \leq t \leq k} \frac{1}{t !} \mid F^{l}\left(v_{j}\right)\left[-\log F^{l}\left(v_{j}\right)\right]^{t}\right. \\
& \left.-\left.G^{l / j}(x)\left[-\log G^{l / j}(x)\right]^{t}\right|^{\theta \wedge 1}\right) I\left[N_{j}=l\right] \\
& \leq \frac{1}{\log n} \sum_{j=1}^{n} \frac{1}{j} E \alpha_{N_{j}}\left(v_{j}\right)+\frac{1}{\log n} \sum_{j=1}^{n} \frac{1}{j} k^{\theta \wedge 1} \max _{0 \leq t \leq k} \frac{1}{t !} \frac{\left|F^{j}\left(v_{j}\right)-G(x)\right|^{\theta \wedge 1}}{G^{2 \theta \wedge 2}(x)} .
\end{aligned}
$$

Because from (7) we have $F\left(v_{n}\right)^{n} \rightarrow G(x)$, thus by Lemma 2

$$
\left|V_{2}(n)\right| \stackrel{\text { a.s. }}{\longrightarrow} 0, \text { as } n \rightarrow \infty \text {. }
$$

Thus (13) is proved in case $G(x)>0$.

Step 2. Let us consider the case $x$ such that $G(x)=0$.

By the part of Theorem proved above and monotonicity the indicator function for arbitrary $\delta>0, x \leq 0$, we have

$$
\begin{aligned}
0 & \leq \frac{1}{\log n} \sum_{j=1}^{n} \frac{1}{j}\left(\xi_{N_{j}, j}(k)-H_{G, k, N_{j} / j}^{\theta}(x)\right)=\frac{1}{\log n} \sum_{j=1}^{n} \frac{1}{j} \xi_{N_{j}, j}(k) \\
& \leq \frac{1}{\log j} \sum_{j=1}^{n} \frac{1}{j} I\left[a_{j} X_{N_{j}-k: N_{j}}+b_{j}<\delta\right] \\
& \leq H_{G, k, \delta_{o}}^{\theta}(\delta)+\frac{1}{\log n} \sum_{j=1}^{n} \frac{1}{j} I\left[\frac{N_{j}}{j}<\delta_{o}\right]+\delta .
\end{aligned}
$$

Then from the arbitrariness of $\delta>0$, Lemma 2(b) and (12), we have (13).

Step 3. We show the proof of (14) because (15) runs similarly.

For every $\epsilon>0$ let us define the partition of real axis $\Pi(\epsilon)=\{-\infty=$ 
$\left.c_{o}<c_{1}<c_{2}<\cdots<c_{m(\epsilon)}=\infty\right\}$ such that $\sup _{x, y \in\left(c_{i}, c_{i+1}\right)}|f(x)-f(y)|<$ $\epsilon / 2, i=0,1,2, \ldots, m(\epsilon)-1$. Let us define $A(x)=s_{i}$, for $x \in\left(c_{i}, c_{i+1}\right), i=$ $0,1,2, . ., m(\epsilon)-1$, where $s_{i}=\sup _{t \in\left(c_{i}, c_{i+1}\right)} f(t), i=0,1,2, \ldots, m(\epsilon)-1$. For a sufficiently large $n$, we have

$$
\begin{aligned}
\frac{1}{\log n} & \sum_{j=1}^{n} \frac{1}{j} f\left(a_{j}\left(X_{N_{j}-k: N_{j}}-b_{j}\right)\right) \leq \frac{1}{\log n} \sum_{j=1}^{n} \frac{1}{j} A\left(a_{j}\left(X_{N_{j}-k: N_{j}}-b_{j}\right)\right) \\
& =\sum_{k=1}^{m(\epsilon)} s_{k} H_{G, k, N_{j} / j}^{\theta}\left(\left(c_{k}, c_{k+1}\right)+\frac{\epsilon}{2}\right. \\
& =\int_{-\infty}^{\infty} A(x) H_{G, k, N_{j} / j}^{\theta}(d x)+\frac{\epsilon}{2} \\
& \leq \int_{-\infty}^{\infty} f(x) H_{G, k, N_{j} / j}^{\theta}(d x)+\int_{-\infty}^{\infty}|A(x)-f(x)| H_{G, k, N_{j} / j}^{\theta}(d x)+\frac{\epsilon}{2} \\
& \leq \int_{-\infty}^{\infty} f(x) H_{G, k, N_{j} / j}^{\theta}(d x)+\epsilon .
\end{aligned}
$$

From the arbitrariness of $\epsilon$, we get (14).

Step 4. Now we will prove the second part of Theorem 1.

If $0<G(x)<1$ then, considering the different cases of limiting laws $G_{1}, G_{2, \alpha}$, and $G_{3, \alpha}$, and taking into account inequality

$$
\left|e^{x}-e^{y}\right| \leq|x-y|\left(e^{x}+e^{y}\right),
$$

we always obtain

$$
\begin{aligned}
\left|G^{\frac{N_{j}}{j}}(x)-G^{\lambda}(x)\right| & \leq\left|\frac{N_{j}}{j}-\lambda\right|\left(\left|e^{-x}\right| \vee\left|x^{-\alpha}\right| \vee\left|x^{\alpha}\right|\left(G^{\frac{N_{j}}{j}}(x)+G^{\lambda}(x)\right)\right) \\
& \leq C\left|\frac{N_{j}}{j}-\lambda\right| .
\end{aligned}
$$

On the other hand, by Lemma 1, we have

$$
\begin{aligned}
& \left|H_{G, k, N_{j} / j}^{\theta}(x)-H_{G, k, \lambda}^{\theta}(x)\right| \leq\left|H_{G, k, N_{j} / j}(x)-H_{G, k, \lambda}(x)\right|^{\theta \wedge 1} \\
& \quad \leq k^{\theta \wedge 1} \max _{0 \leq t \leq k}\left|G^{N_{j} / j}(x)\left[-\log G^{N_{j} / j}(x)\right]^{t}-G^{\lambda}(x)\left[-\log G^{\lambda}(x)\right]^{t}\right|^{\theta \wedge 1} \\
& \quad \leq C \max _{0 \leq t \leq k}\left|G^{N_{j} / j}(x)-G^{\lambda}(x)\right|^{(1-\beta t)(\theta \wedge 1)} \\
& \quad \leq C d\left(N_{j} / j, \lambda\right)^{(1-\beta k)(\theta \wedge 1)} \wedge 1,
\end{aligned}
$$

for every $0<\beta<1 / k$, which, by Lemma 2(b), proves Theorem 1 . For $G_{2, \alpha}(x)=0$ or $G_{3, \alpha}(x)=1$ the proof of the second part of Theorem 1 is obvious. 


\section{Examples and applications}

\section{Example 1.}

(a) Let $\left\{N_{j}, j \geq 1\right\}$ be a sequence of independent random variables such that $N_{j} \sim \beta_{j}+\gamma_{j}$ Pois $\left(\lambda_{j}\right)$ (the uncentred and unnormalized Poisson's law, $\left.P\left[N_{j}=\beta_{j}+k \gamma_{j}\right]=\frac{\lambda_{j}^{k}}{k !} e^{-\lambda_{j}}, k=0,1,2, \ldots\right)$ for some sequence of nonnegative numbers $\left\{\lambda_{j}, \gamma_{j}, j \geq 1\right\}$ and a sequence of numbers $\left\{\beta_{j}, j \geq 1\right\}$. If

$$
\beta_{j}+\gamma_{j} \lambda_{j}=O(j),
$$

then (9) holds. On the other hand, if for some $\delta_{o}>0, \mu>0$, we have

$$
\frac{\left(j \delta_{o}-\beta_{j}\right)(\log j)^{\mu}}{\gamma_{j} \sqrt{\lambda_{j}}} \leq C,
$$

then (12) holds.

(b) Let $\left\{N_{j}, j \geq 1\right\}$ be a sequence of independent random variables such that $N_{j} \sim \beta_{j}+\gamma_{j} B\left(n_{j}, p_{j}\right)$ (the uncentred and unnormalized Bernouilly's law, $\left.P\left[N_{j}=\beta_{j}+\gamma_{j} k\right]=\left(\begin{array}{c}n_{j} \\ k\end{array}\right) p_{j}^{k}\left(1-p_{j}\right)^{n_{j}-k}, k=0,1,2, \ldots, n_{j}\right)$ for some sequence of nonnegative numbers $\left\{n_{j}, \gamma_{j}, j \geq 1\right\}$, numbers $\left\{\beta_{j}, j \geq 1\right\}$ and numbers $\left\{p_{j}, j \geq 1\right\}$ such that $0 \leq p_{j} \leq 1, j \geq 1$. If

$$
\beta_{j}+\gamma_{j} n_{j} p_{j}=O(j)
$$

then (9) holds. On the other hand, if for some $\delta_{o}>0, \mu>0$, we have

$$
\frac{\left(j \delta_{o}-\beta_{j}\right)(\log j)^{\mu} \sqrt{n_{j} p_{j}\left(1-p_{j}\right)}}{\gamma_{j}} \leq C,
$$

then (12) holds.

(c) Let $\left\{N_{j}, j \geq 1\right\}$ be a sequence of independent random variables such that $N_{j} \sim \beta_{j}+\gamma_{j} U\left(n_{j}\right)$ (the uncentred and unnormalized uniform law, $\left.P\left[N_{j}=\beta_{j}+\gamma_{j} k\right]=\frac{1}{n_{j}}, k=1,2, \ldots, n_{j}\right)$ for some sequence of nonnegative numbers $\left\{n_{j}, \gamma_{j}, j \geq 1\right\}$ and sequence of numbers $\left\{\beta_{j}, j \geq 1\right\}$. If

$$
\beta_{j}+\gamma_{j} \frac{n_{j}+1}{2}=O(j)
$$

then (9) holds. On the other hand, if for some $\delta_{o}>0, \mu>0$, we have

$$
\frac{\left(j \delta_{o}-\beta_{j}\right)(\log j)^{\mu}}{n_{j} \gamma_{j}} \leq C,
$$

then (12) holds.

Proof of Example 1 (a). Under such defined sequence $\left\{N_{j}, j \geq 1\right\}$ we have $E N_{j}=\beta_{j}+\gamma_{j} \lambda_{j}, j \geq 1$, and

$$
\sum_{k=1}^{N} \sum_{j=1}^{k-1} \frac{1}{j k} E\left(\frac{N_{j} \wedge N_{k}}{k} \wedge 1\right) \leq \sum_{k=1}^{N} \sum_{j=1}^{k-1} \frac{\beta_{j}+\gamma_{j} \lambda_{j}}{j k^{2}} \leq \sum_{k=1}^{N} \frac{1}{k}=O\left((\log N)^{2-\mu}\right) .
$$


Furthermore, it is easy to check that for arbitrary $\lambda>0$, we have

$$
\sup _{k \geq 0}\left\{\frac{\lambda^{k}}{k !} e^{-\lambda}\right\} \leq \max \left\{\frac{\lambda^{[\lambda]}}{[\lambda] !} e^{-\lambda}, \frac{\lambda^{[\lambda]+1}}{([\lambda]+1) !} e^{-\lambda}\right\},
$$

and by Stirling formulae we have

$$
\sup _{k \geq 0}\left\{\frac{\lambda^{k}}{k !} e^{-\lambda}\right\} \leq \frac{C}{\sqrt{2 \pi \lambda}}
$$

Thus

$$
P\left[\frac{N_{n}}{n}<\delta_{0}\right]=\sum_{k=0}^{\left(\delta_{o} j-\beta_{j}\right) / \gamma_{j}} \frac{\lambda_{j}^{k}}{k !} e^{-\lambda_{j}} \leq C \frac{\delta_{o} j-\beta_{j}}{\gamma_{j} \sqrt{\lambda_{j}}}, \quad j \geq 1,
$$

which ends the proof of point (a). The proof of points (b)-(c) is similar and will be omitted.

The different constructions of stationary sequences nonidentically distributed random variables $\left\{X_{n}, n \geq 1\right\}$, satisfying conditions $\alpha_{j}\left(v_{j}\right) \rightarrow 0$ or $\alpha_{j, h}\left(v_{j}, v_{h}\right) \rightarrow 0$ as $j, h \rightarrow \infty$ may be found in [11].

\section{References}

[1] Aksomaitis, A., Transfer theorems in a max-scheme, Litovsk. Mat. Sb. 29 (2) (1989), 207-211 (Russian).

[2] Barndorff-Nielsen, O., On the limit distribution of the maximum of a random number of independent random variables, Acta. Math. Acad. Sci. Hungar. 11 (1964), 399-403.

[3] Berkes, I., Csáki, E., A universal result in almost sure central limit theory, Stoch. Proc. Appl. 94 (2001), 105-134.

[4] Brosamler, G. A., An almost everywhere central limit theorem, Math. Proc. Cambridge Philos. Soc. 104 (1988), 561-574.

[5] Berman, S. M., Limiting distribution of the maximum in the sequence of dependent random variables, Ann. Math. Statist. 33 (1962), 894-908.

[6] Cheng, S., Peng, L., Qi, Y., Almost sure convergence in extreme value theory, Math. Nachr. 190 (1998), 43-50.

[7] Fahrner, I., Almost Sure Versions of Weak Limit Theorems, Shaker Verlag, Aachen, 2000.

[8] Fahrner, I., Stadmüller, U., On almost sure max-limit theorems, Statist. Probab. Lett. 37 (1998), 229-236.

[9] Galambos, J., The Asymptotic Theory of Extreme Order Statistics, Wiley Series in Probability and Mathematical Statistics, John Wiley \& Sons, New York-ChichesterBrisbane, 1978.

[10] Hörmann, S., An extension of almost sure central limit theory, Statist. Probab. Lett. 76 (2006), 191-202.

[11] Jakubowski, A., Asymptotic Independent Representations for Sums and Order Statistics of Stationary Sequences, NCU Press publications, Torun, 1991.

[12] Krajka, A., Wasiura, J., On the almost sure central limit theorem for randomly indexed sums, Math. Nachr. 282 (4) (2009), 569-580.

[13] Lacey, M. T., Philipp, W., A note on the almost sure central limit theorem, Statist. Probab. Lett. 9 (1990), 201-205. 
[14] Leadbetter, M. R., Lindgren, G., Rootzen, H., Extremes and Related Properties of Random Sequences and Processes, Springer, Berlin, 1983.

[15] O'Brien, G. L., The maximum term of uniformly mixing stationary process, Z. Wahr. verw. Gebiete 30 (1974), 57-63.

[16] Robbins, H., The asymptotic distribution of the sums of a random number of random variables, Bull. Amer. Math. Soc. 54 (1948), 1151-1161.

[17] Resnick, S. I., Extreme Values. Regular Variation and Point Processes, Springer, 1987.

[18] Stadtmüller, U., Almost sure versions of distributional limit theorems for certain order statistics, Statist. Probab. Lett. 58 (2002), 413-426.

[19] Schatte, P., On strong version of the central limit theorem, Math. Nachr. 137 (1988), $249-256$.

Andrzej Krajka

Maria Curie-Skłodowska University

Pl. Marii Curie-Skłodowskiej 1

20-031 Lublin

Poland

e-mail: andrzej.krajka@umcs.lublin.pl
Zdzisław Rychlik

Maria Curie-Skłodowska University

Pl. Marii Curie-Skłodowskiej 1

20-031 Lublin

Poland

e-mail: rychlik@hektor.umcs.lublin.pl

Joanna Wasiura-Maślany

The John Paul II Catholic University of Lublin

Aleje Racławickie 14

20-950 Lublin

Poland

e-mail: jwaaa@wp.pl

Received November 14, 2019 